\title{
ARTICLE OPEN \\ Enhanced thermal stability of organic solar cells comprising ternary D-D-A bulk-heterojunctions
}

\author{
Dominik Landerer ${ }^{1,2}$, Adrian Mertens ${ }^{1,2}$, Dieter Freis ${ }^{1}$, Robert Droll $^{1}$, Tobias Leonhard ${ }^{1,2}$, Alexander Deniz Schulz ${ }^{1}$, Daniel Bahro ${ }^{1,2}$ and \\ Alexander Colsmann (iD) ${ }^{1,2}$
}

Ternary absorber blends have recently been identified as promising concepts to spectrally broaden the absorption of organic bulkheterojunction solar cells and hence to improve their power conversion efficiencies. In this work, we demonstrate that D-D-A ternary blends comprising two donor polymers and the acceptor $\mathrm{PC}_{61} \mathrm{BM}$ can also significantly enhance the thermal stability of the solar cell. Upon harsh thermal stress at $120^{\circ} \mathrm{C}$ for $2 \mathrm{~h}$, the ternary solar cells show only a minor relative deterioration of $10 \%$. Whereas the polymer/fullerene blend PTB7-Th:PC ${ }_{61} \mathrm{BM}$ is rather unstable under these conditions, its degradation was efficiently suppressed by incorporating the near infrared-absorbing polymer PDTP-DFBT. Spectroscopic ellipsometry investigations and an effective medium analysis of the ternary absorber blend revealed that the domain conformation in presence of PDTP-DFBT remains

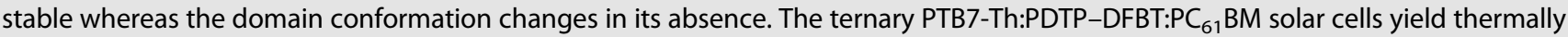
stable power conversion efficiencies of up to $6 \%$.

npj Flexible Electronics (2017)1:11; doi:10.1038/s41528-017-0011-z

\section{INTRODUCTION}

The continuous improvement of materials and device architectures for organic bulk-heterojunction solar cells nowadays constantly enables power conversion efficiencies (PCEs) beyond $10 \%{ }^{1,2}$ While the PCEs of state-of-the-art organic solar cells are sufficient to meet the demands of some first applications and hence to foster market entry, the device stability often drags behind. The stability of organic solar cells is often limited by a metastable bulk-heterojunction morphology, the diffusion of electrode or buffer layer components into adjacent layers, material decomposition through oxygen and water ingress, irradiation, mechanical stress or heat. ${ }^{3-5}$ Yet, excellent thermal stability of the organic solar cells is a prerequisite for most future applications. Besides thermal stress when the solar cell is exposed to the sun, the devices may have to endure harsh conditions during fabrication, e.g., when manufacturing car roofs, tiles or façade elements, typically requiring lamination temperatures of $120^{\circ} \mathrm{C}$ for several hours. 6,7

Upon thermal stress, one of the major degradation mechanisms is the aggregation of fullerene acceptors, commonly $[6,6]$-phenyl $\mathrm{C}_{71}$-butyric acid methyl ester $\left(\mathrm{PC}_{71} \mathrm{BM}\right)$ or $[6,6]$-phenyl $\mathrm{C}_{61}$-butyric acid methyl ester $\left(\mathrm{PC}_{61} \mathrm{BM}\right){ }^{8}{ }^{8}{ }^{9}$ In the recent literature, several methods were discussed to hamper fullerene aggregation-for example sample irradiation during annealing, ${ }^{10}$ structural modification of fullerenes ${ }^{11}$ or replacing fullerenes with non-fullerene acceptors. ${ }^{12}$ Another promising concept to reduce degradation in fullerene-based solar cells is the use of third components which can improve the mechanical, photo, air and thermal stability of organic solar cells. ${ }^{13}$ Such third components can be crosslinkers, ${ }^{14-16}$ conventional fullerenes, ${ }^{17-19}$ modified fullerene derivatives, $^{20-22}$ compatibilizers $^{23,24}$ or insulating polymers. ${ }^{25}$
In this work, we overcome the aggregation of the fullerene acceptor $\mathrm{PC}_{61} \mathrm{BM}$ during thermal annealing by employing ternary D-D-A blends comprising two commercially available donor polymers. After thermal stress at $120^{\circ} \mathrm{C}$ for $2 \mathrm{~h}$, the corresponding solar cells maintain over $90 \%$ of their initial performance. The microscopic domain conformation was monitored by atomic force microscopy and advanced spectroscopic ellipsometry.

\section{RESULTS AND DISCUSSIONS}

Choice of materials

Being one of the "fruit-flies" of modern organic photovoltaics, we deliberately investigated the thermal stability of solar cells comprising the wide-bandgap donor polymer poly[[4,8-bis[5-(2ethylhexyl)thiophen-2-yl]benzo[1,2-b:4,5-b']dithiophene-2,6-diyl] [3-fluoro-2-[(2-ethylhexyl)carbonyl]thieno[3,4-b]thiophenediyl]] (PTB7-Th) to enable comparison with previous literature reports. ${ }^{26}$ Advanced solar cells comprising PTB7-Th can exhibit PCEs beyond $10 \%{ }^{27}$ but the thermal stability of PTB7-Th solar cells and solar cells of its derivatives is moderate. ${ }^{10,19}$ On the other hand, we studied the thermal stability of solar cells comprising the narrowbandgap donor polymer poly[[2,7-(5,5-bis-(3,7-dimethyloctyl)-5Hdithieno[3,2-b:2', 3'-d]pyran)-alt-4,7-(5,6-difluoro-2,1,3-benzothiadiazole)]] (PDTP-DFBT), which is often employed to efficiently harvest near-infrared light. $^{28,} 29$ Initially, both donor polymers were investigated in binary bulk-heterojunctions using the acceptor $\mathrm{PC}_{61} \mathrm{BM}$. We intentionally opted for $\mathrm{PC}_{61} \mathrm{BM}$ rather than $P C_{71} B M$ as it is the industrially more relevant, yet less stabilizing, fullerene. For example, certain binary bulk-heterojunctions were reported to be stabilized by $P C_{71} B M$ alone, whereas use of $P C_{61} B M$ leads to rapid device failures. ${ }^{30}$

\footnotetext{
${ }^{1}$ Light Technology Institute, Karlsruhe Institute of Technology (KIT), Engesserstrasse 13, 76131 Karlsruhe, Germany and ${ }^{2}$ Material Research Center for Energy Systems (MZE), Karlsruhe Institute of Technology (KIT), Strasse am Forum 7, 76131 Karlsruhe, Germany

Correspondence: Alexander Colsmann (alexander.colsmann@kit.edu)
}

Received: 17 March 2017 Revised: 3 August 2017 Accepted: 21 August 2017

Published online: 04 December 2017 
(a)

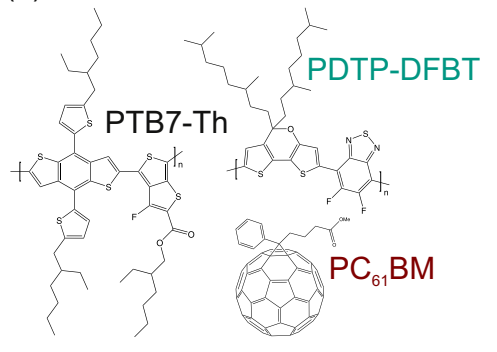

(b)

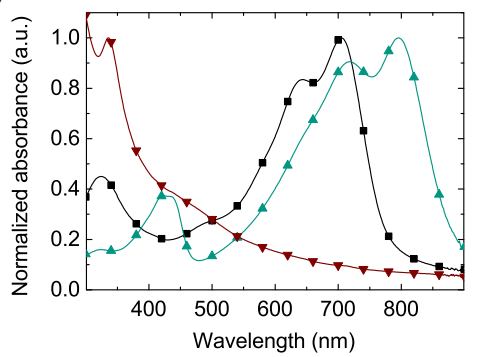

(c)

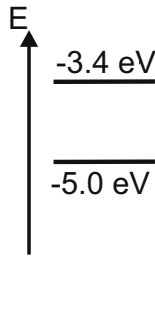

(d)

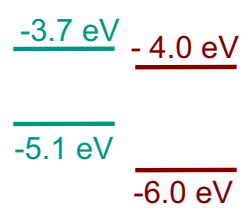

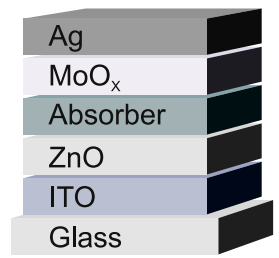

Fig. 1 a Chemical structures of the light-harvesting organic components PBT7-Th, PDTP-DFBT and PC ${ }_{61} B M$. b UV-Vis absorption spectra of thin-films. For better visibility, the spectra are normalized to their maximum. c Diagram of the relevant charge carrier transport energies: $\mathrm{HOMO}$ energies were estimated by PESA, LUMO energies were estimated from HOMO energies and $E_{\mathrm{g}}$. d Solar cell device architecture

The chemical structures of all components are depicted in Fig. $1 \mathrm{a}$, and their ultraviolet-visible (UV-Vis) absorption spectra (thinfilms) are shown in Fig. 1b. With an optical bandgap of $E_{\mathrm{g}}=1.6 \mathrm{eV}$ (calculated from long-wavelength absorbance onset), PTB7-Th absorbs mainly between $500-750 \mathrm{~nm}$. Using photoelectron yield spectroscopy in air (PESA), we estimated its highest occupied molecular orbital $(\mathrm{HOMO})$ energy to $E_{\mathrm{HOMO}}=-5.0 \mathrm{eV}$ (Fig. S1a, Suppl. Information). The corresponding lowest unoccupied molecular orbital (LUMO) energy was estimated $E_{\mathrm{LUMO}}=-3.4$ $\mathrm{eV}$ from the HOMO and $E_{\mathrm{g}}$. PDTP-DFBT mainly absorbs at 650-850 $\mathrm{nm}$ and exhibits an additional small absorption peak around $420 \mathrm{~nm}$. We found an optical bandgap $E_{\mathrm{g}}=1.4 \mathrm{eV}, \mathrm{a}$ $\mathrm{HOMO}$ energy $E_{\mathrm{HOMO}}=-5.1 \mathrm{eV}$ (Fig. S1b, Suppl. Information) and a LUMO energy $E_{\mathrm{LUMO}}=-3.7 \mathrm{eV}$. $\mathrm{PC}_{61} \mathrm{BM}$ mainly absorbs between 300 and $500 \mathrm{~nm}$, the bandgap is $E_{\mathrm{g}}=2.0 \mathrm{eV}$, and its $\mathrm{HOMO}$ and LUMO energies are $E_{\mathrm{HOMO}}=-6.0 \mathrm{eV}$ (Fig. S1C, Suppl. Information) and $E_{\mathrm{LUMO}}=-4.0 \mathrm{eV}$. The relevant energy levels of all three components are summarized in Fig. 1c.

All polymer:fullerene absorber blends $(1: 1.5 \mathrm{w} / \mathrm{w})$ were deposited from $o$-xylene using the co-solvent $p$-anisaldehyde ${ }^{30}$ and incorporated into the inverted device architecture glass/ITO/ZnO/ absorber/molybdenum oxide $\left(\mathrm{MoO}_{\mathrm{x}}\right) /$ silver $(\mathrm{Ag})$ as depicted in Fig. 1d. Importantly, the co-solvent $p$-anisaldehyde exhibits a comparatively low boiling point, ensuring its complete evaporation after thin-film deposition, ${ }^{31}$ whereas common other co-solvents such as 1,8-diiodooctane may partly remain within the bulkheterojunction where they may accelerate device deterioration. ${ }^{30}$ To investigate the thermal stability of the solar cells, we annealed the samples at $120^{\circ} \mathrm{C}$ for $2 \mathrm{~h}$, which is often considered the critical condition for industrial relevant lamination processes for consumer products. ${ }^{6,7}$

Binary polymer:fullerene blends and their thermal stability The current density-voltage $(J-V)$ curves of PTB7-Th:PC ${ }_{61}$ BM solar cells are depicted in Fig. 2a, and their key performance parameters (short-circuit current density $J_{s c}$ fill factor FF, open-circuit voltage $V_{\text {oc }}$ and PCE) are summarized in Table 1.

After annealing of the devices $\left(120^{\circ} \mathrm{C}, 2 \mathrm{~h}\right)$, the PCE of the PTB7Th: $\mathrm{PC}_{61} \mathrm{BM}$ solar cells decreased by $30 \%$ (rel.), i.e., a drop from 7.4 to $5.2 \%$. Although the $V_{\text {oc }}$ slightly improved upon thermal stress, which may be attributed to the diffusion of the fullerenes to the transport layer interface during annealing ${ }^{30}$ or a better phase separation and purer material domains after annealing, ${ }^{1}$ the reduced $\mathrm{FF}$ and $J_{\mathrm{sc}}$ caused significant device deterioration. To investigate the origin of the lower $J_{\mathrm{sc}}$ after thermal annealing, we recorded the external quantum efficiency (EQE) of the solar cell. The annealing process dramatically reduces the EQE of the PTB7Th:PC ${ }_{61} B M$ solar cell over the whole spectrum (Fig. S2a, Suppl. Information). Figure $2 \mathrm{~b}$ depicts the EQEs before and after sample annealing, normalized to their maximum amplitude. The stronger
EQE deterioration below $550 \mathrm{~nm}$ indicates that the degradation was mainly driven by the fullerene.

Fullerenes are known to aggregate under thermal stress. Larger fullerene domains can lead to declined exciton dissociation since photo-generated excitons on the fullerenes may not reach an interface within their lifetime. Indeed, in atomic force micrographs (Fig. 2c), we find foot prints of large fullerene domains on the sample surface, i.e., sharp peaks with a maximal height $R_{\max }=74.8$ $\mathrm{nm}$ (surface roughness $R_{\mathrm{rms}}=1.2 \mathrm{~nm}$ before and $2.4 \mathrm{~nm}$ after annealing). We note that replacing $\mathrm{PC}_{61} \mathrm{BM}$ by $\mathrm{PC}_{71} \mathrm{BM}$ resulted in an even stronger degradation with a decrease in PCE of more than $30 \%$ (rel.), since $\mathrm{PC}_{71} \mathrm{BM}$ contributes even more to the device's photo current ${ }^{19}$ (Fig. S3, Suppl. Information).

For reference, we also studied the degradation of PTB7-Th: $\mathrm{PC}_{61} \mathrm{BM}$ solar cells that were fabricated omitting the co-solvent $p$ anisaldehyde (Fig. 2a). On a lower efficiency level, we observed the same $30 \%$ (rel.) performance degradation upon thermal annealing, indicating that the co-solvent does not influence the observed device deterioration.

Employing PDTP-DFBT:PC ${ }_{61} \mathrm{BM}$ to harvest sunlight draws a completely different picture: remarkably, after thermal annealing for $2 \mathrm{~h}$ at $120^{\circ} \mathrm{C}$, the solar cells' key performance parameters remained nearly constant (Fig. 2d, Table 1). Again, the $V_{\text {oc }}$ slightly increases. However, this time, FF and $J_{\mathrm{sc}}$ as well as the EQE (Fig. S2C, Suppl. Information) showed hardly any of the deterioration that we observed in PTB7-Th:PC ${ }_{61}$ BM solar cells before. Normalized to its maximum, in Fig. $2 \mathrm{e}$, the $\mathrm{EQE}$ shows only a marginally enhanced degradation in the fullerene's spectral regime. Accordingly, annealing had only minor effect on the surface of the bulkheterojunction, as visible in the atomic force micrographs in Fig. 2f. Although the surface roughness of PDTP-DFBT:PC ${ }_{61} B M$ absorber layers is somewhat higher $\left(R_{\mathrm{rms}}=4.6 \mathrm{~nm}\right.$ before and $4.8 \mathrm{~nm}$ after annealing) than the surface roughness of PTB7-Th: $\mathrm{PC}_{61} \mathrm{BM}$ photo-active layers, we find only a minor increase of the relative maximal height of a few nanometer. We observed none of the sharp peaks that we observed in PTB7-Th:PC ${ }_{61} B M$ after annealing. This indicates an almost completely suppressed aggregation of $\mathrm{PC}_{61} \mathrm{BM}$ in PDTP-DFBT. This outstanding thermal stability may originate from a better miscibility of PDTP-DFBT and $\mathrm{PC}_{61} \mathrm{BM}$ versus PTB7-Th and $\mathrm{PC}_{61} \mathrm{BM}$, and hence a more favorable conformation of the fullerene domains, ${ }^{11,30}$ or from a higher glass transition temperature $T_{\mathrm{g}}$ of PDTP-DFBT. ${ }^{32}$

Optoelectronic properties of ternary blends

PDTP-DFBT suppresses the aggregation of fullerenes in binary bulk-heterojunctions and hence obtains thermally stable organic solar cells. In the next step, we explored whether PDTP-DFBT can also stabilize PTB7-Th:PC ${ }_{61}$ BM bulk-heterojunctions that, left alone, are not thermally stable. 

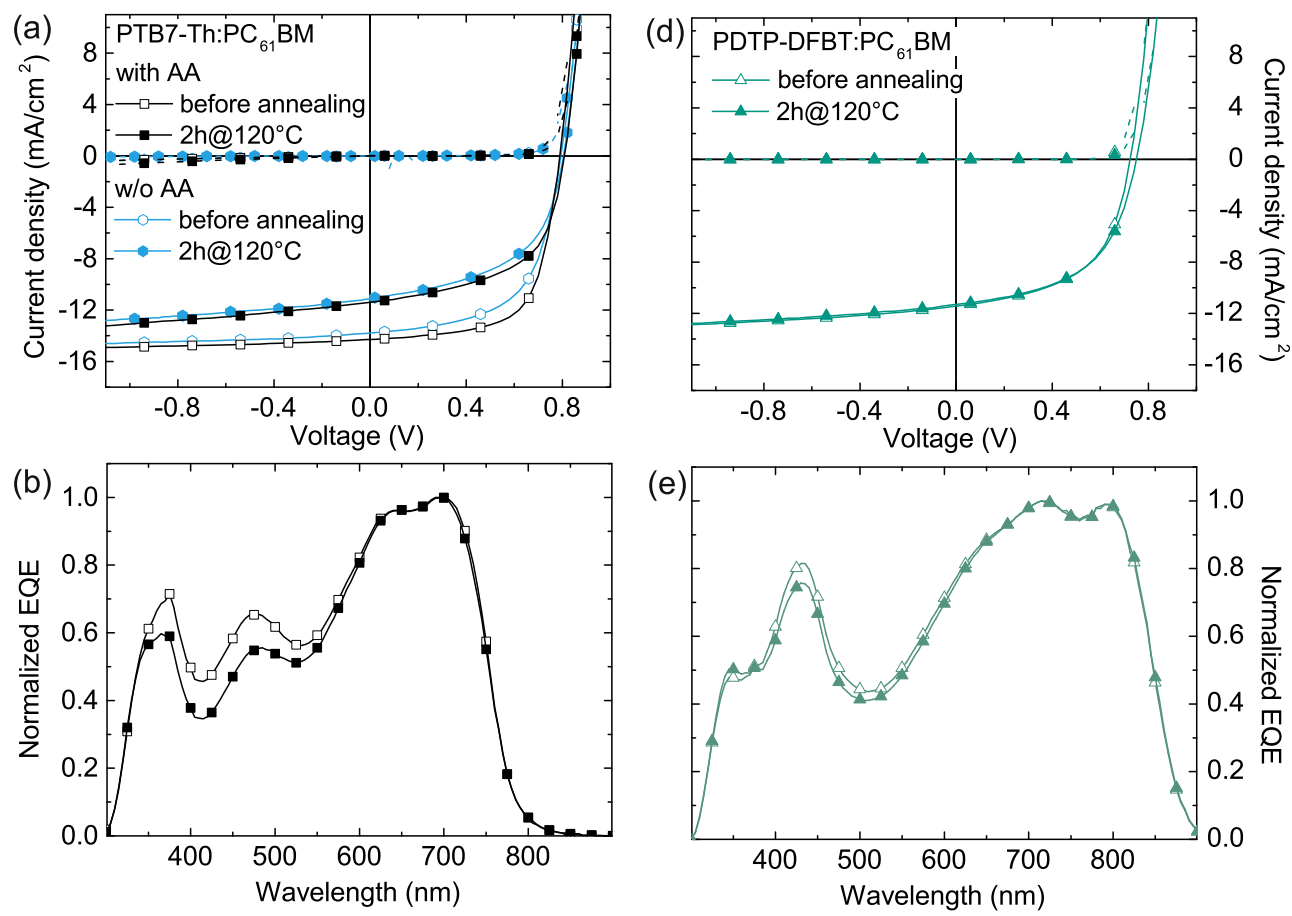

(c)

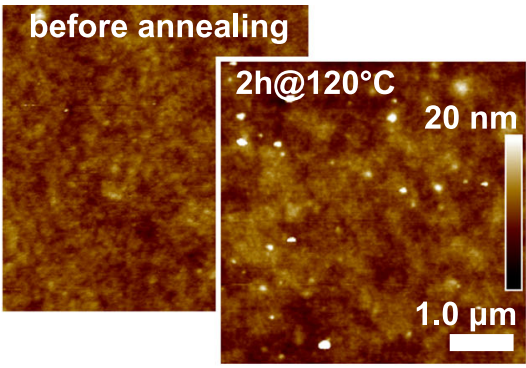

(f)

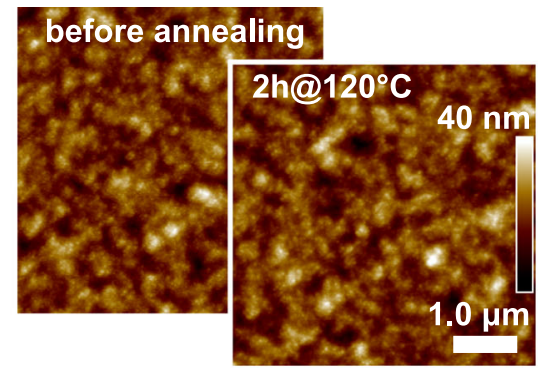

Fig. 2 a Typical $J-V$ curves of binary PTB7-Th:PC ${ }_{61} B M$ solar cells under AM1.5 illumination (solid lines) and in the dark (dashed lines), processed after adding $2 \% p$-anisaldehyde (AA) to the stock solution (black) and processed omitting the co-solvent (blue). The samples were measured before annealing (open symbols) and after thermal annealing $\left(120^{\circ} \mathrm{C}, 2 \mathrm{~h}\right.$, closed symbols). b Corresponding normalized external quantum efficiencies. 1 out of 5 data points is marked with a symbol to guide the eye. c Topography images of the binary PTB7-Th:PC $61 \mathrm{BM}$ blends processed with AA before (back) and after annealing (front). d-f Same data set for solar cells comprising binary PDTP-DFBT:PC ${ }_{61}$ BM absorber layers

Therefore, we successively increased the PDTP-DFBT content $x$ and reduced the PTB7-Th content $1-x$ with an overall constant polymer:fullerene ratio $(1: 1.5 \mathrm{w} / \mathrm{w})$, and studied the optoelectronic device properties as well as their thermal stability. The key performance parameters of the respective solar cells comprising non-annealed PTB7-Th:PDTP-DFBT:PC ${ }_{61} B M \quad$ ([1-x]:x:1.5 w/w/w) absorber layers versus PDTP-DFBT content $x$ are depicted in Fig. $3 a$. Figure $3 \mathrm{~b}$ shows the corresponding EQEs of the solar cells. Starting from the binary PTB7-Th:PC ${ }_{61} \mathrm{BM}$ absorber layer, $J_{\mathrm{sc}}=14$ $\mathrm{mA} / \mathrm{cm}^{2}$ remains constant up to a PDTP-DFBT concentration of $x$ $=40 \%$. In this regime, the EQE reduction in the PTB7-Th absorption regime is compensated by the EQE enhancement in the long-wavelength PDTP-DFBT absorption regime. Above $x=$ $40 \%$, the EQE enhancement caused by PTPD-DFBT is insufficient to compensate the EQE reduction in the PTB7-Th absorption regime. Consequently, the $J_{s c}$ of the ternary absorber blend decreases and eventually reproduces the $J_{s c}$ of the binary PTPD-DFBT:PC ${ }_{61} B M$ solar cells. Despite the spectrally broadened absorption, ternary PTB7-Th:PDTP-DFBT:PC ${ }_{61}$ BM blends do not generate more photocurrent $\left(J_{s c}\right)$ than the binary PTB7-Th:PC ${ }_{61} \mathrm{BM}$ solar cells. Likewise, the FF decreases from 66 to $55 \%$ upon increasing the PDTP-DFBT content in the absorber blend. Together with the nearly linear reduction in $V_{\mathrm{OC}}$, the PCE of solar cells comprising ternary blends is constrained between the binary extremes, PCE $=7.4 \%$ at $x=0 \%$ and $4.5 \%$ at $x=100 \%$.

Commonly, ternary absorber blends are classified in two categories: (i) In a parallel-like bulk-heterojunction, holes are generated and transported individually on the two polymer domains without any notable interaction. ${ }^{33-35}$ (ii) Two components (here: two donor polymers) form an organic alloy with strong physical and electronic interaction. ${ }^{36-39}$ To classify the ternary absorber PTB7-Th:PDTP-DFBT:PC ${ }_{61} B M$, we investigated the interaction of PTB7-Th and PDTP-DFBT by photoluminescence $(\mathrm{PL})$ spectroscopy on PTB7-Th:PDTP-DFBT blend films omitting $\mathrm{PC}_{61} \mathrm{BM}$. In the PL spectra in Fig. 3c, neat PTB7-Th exhibits a broad emission peak from 720 to $850 \mathrm{~nm}$, which overlaps well with the absorption of PDTP-DFBT (see Fig. 1 for PDTP-DFBT absorption). Upon mixing PDTP-DFBT into PTB7-Th, the PL signal of PDTP-DFBT increases continuously while the emission of PTB7Th is efficiently quenched, providing evidence for energy transfer from PTB7-Th to PDTP-DFBT. Hence we conclude that the PTB7Th:PDTP-DFBT:PC ${ }_{61} B M$ blend is a type (ii) system. 
Table 1. Key performance parameters of solar cells comprising PTB7-Th:PDTP-DFBT:PC ${ }_{61} B M([1-x]: x: 1.5 \mathrm{w} / \mathrm{w} / \mathrm{w})$ light harvesting layers before and after thermal annealing $\left(120^{\circ} \mathrm{C}, 2 \mathrm{~h}\right)$. The data set represents more than 20 samples per entry

\begin{tabular}{|c|c|c|c|c|c|c|c|c|c|}
\hline PTB7-Th:PDTP-DFBT:PC ${ }_{61}$ BM & annealing & $J_{\mathrm{SC}}\left(\mathrm{mA} / \mathrm{cm}^{2}\right)$ & $V_{O C}(\mathrm{mV})$ & $\mathrm{FF}(\%)$ & PCE (\%) & $\mathrm{PCE}^{\mathrm{a}}(\%)$ & $\mathrm{PCE} / \mathrm{PCE}_{0}^{\mathrm{b}}(\%)$ & $R_{\mathrm{rms}}{ }^{\mathrm{c}}(\mathrm{nm})$ & $R_{\max }{ }^{\mathrm{d}}(\mathrm{nm})$ \\
\hline 1.0:0.0:1.5 (w/o AA) & $2 \mathrm{~h} @ 120^{\circ} \mathrm{C}$ & $11.2 \pm 0.1$ & $805 \pm 3$ & $51 \pm 2$ & $4.6 \pm 0.1$ & 4.8 & & 1.8 & 18.7 \\
\hline 1.0:0.0:1.5 & $2 \mathrm{~h} @ 120^{\circ} \mathrm{C}$ & $11.4 \pm 0.4$ & $808 \pm 4$ & $52 \pm 1$ & $5.2 \pm 0.2$ & 5.5 & & 2.4 & 74.8 \\
\hline \multirow[t]{2}{*}{$0.8: 0.2: 1.5$} & w/o & $14.1 \pm 0.5$ & $767 \pm 6$ & $65 \pm 2$ & $7.0 \pm 0.2$ & 7.5 & 83 & 1.3 & 12.2 \\
\hline & $2 \mathrm{~h} @ 120^{\circ} \mathrm{C}$ & $12.8 \pm 0.3$ & $792 \pm 2$ & $58 \pm 2$ & $5.8 \pm 0.2$ & 6.1 & & 1.4 & 16.9 \\
\hline \multirow[t]{2}{*}{$0.4: 0.6: 1.5$} & w/o & $13.4 \pm 0.4$ & $736 \pm 5$ & $61 \pm 1$ & $6.0 \pm 0.2$ & 6.5 & 93 & 2.4 & 18.7 \\
\hline & $2 \mathrm{~h} @ 120^{\circ} \mathrm{C}$ & $12.9 \pm 0.2$ & $759 \pm 4$ & $57 \pm 1$ & $5.6 \pm 0.1$ & 5.8 & & 2.9 & 28.0 \\
\hline \multirow[t]{2}{*}{$0.2: 0.8: 1.5$} & w/o & $12.5 \pm 0.5$ & $722 \pm 3$ & $57 \pm 1$ & $5.1 \pm 0.2$ & 5.4 & 98 & 3.1 & 21.4 \\
\hline & $2 \mathrm{~h} @ 120^{\circ} \mathrm{C}$ & $12.0 \pm 0.5$ & $748 \pm 2$ & $56 \pm 1$ & $5.0 \pm 0.2$ & 5.2 & & 3.3 & 25.1 \\
\hline $0.0: 1.0: 1.5$ & w/o & $11.4 \pm 0.4$ & $724 \pm 8$ & $55 \pm 1$ & $4.5 \pm 0.3$ & 4.9 & 100 & 4.8 & 38.4 \\
\hline
\end{tabular}

We note that the gradual change of $V_{\text {oc }}$ towards the $V_{\text {oc }}$ of the binary blends $(x=0 \%$ or $x=100 \%)$ is often observed in type (i) ternary blends. Yet, this phenomenon has occasionally also been observed in type (ii) ternary material systems $\mathrm{s}^{33,37,40,41}$ and can be caused by an electronic alloy charge transfer state ${ }^{38}$ or by two mixed distinct charge transfer states. ${ }^{39}$

\section{Thermal stability of ternary blends}

To investigate the thermal stability of the ternary PTB7-Th: PDTP-DFBT:PC ${ }_{61}$ BM blend at different PDTP-DFBT concentrations, we thermally stressed all samples discussed previously at $120^{\circ} \mathrm{C}$ for $2 \mathrm{~h}$. The respective device performance data are depicted in Fig. $3 a$ and is summarized in Table 1 . We found that the $J_{\text {sc }}$ of the thermally annealed devices increased towards a PDTP-DFBT concentration $x=40 \%$, where it almost matched the $J_{s c}$ of the devices before annealing. Beyond $x=40 \%, J_{s c}$ decreased, eventually yielding the $J_{s c}$ of binary PDTP-DFBT:PC ${ }_{61}$ BM solar cells. With the FF being rather constant and the $V_{\text {oc }}$ showing the same behavior as in the non-annealed devices, the PCE followed the $J_{s c}$ trend. Under the harsh thermal stress conditions employed in here $\left(120^{\circ} \mathrm{C}, 2 \mathrm{~h}\right)$, we found optimum device performance and stability at $x=40 \%$, i.e., PTB7-Th:PDTP-DFBT:PC ${ }_{61}$ BM (0.6:0.4:1.5) solar cells. Figure 4a specifically compares the $J-V$ curves of the PTB7-Th: PDTP-DFBT:PC ${ }_{61} B M \quad(0.6: 0.4: 1.5)$ solar cells before and after annealing. Again, the $V_{\text {oc }}$ improved slightly from $749 \mathrm{mV}$ before to $777 \mathrm{mV}$ after thermal stressing. The FF declined from 62 to $57 \%$ but still surpasses binary PDTP-DFBT:PC ${ }_{61} B M$. After annealing, we obtained $J_{\mathrm{sc}}=13.4 \mathrm{~mA} / \mathrm{cm}^{2}$ which remained close to the $J_{\mathrm{sc}}=14.2$ $\mathrm{mA} / \mathrm{cm}^{2}$ before annealing-a relative loss of only $6 \%$. Most remarkably, after thermal annealing for $2 \mathrm{~h}$ at $120^{\circ} \mathrm{C}$, the PCE of the ternary solar cells still yielded $6 \%$, generating $15 \%$ more photocurrent than the binary PTB7-Th:PC ${ }_{61} \mathrm{BM}$ solar cell. This stabilization of the device performance is also reflected in the EQE measurements in Fig. 4b. The EQE losses upon annealing all over the spectral regime are far less pronounced than in the binary PTB7-Th:PC ${ }_{61} B M$ mixture and rather resemble the magnitude of relative losses observed in the binary PDTP-DFBT:PC ${ }_{61} B M$ absorber (Fig. 2). We observed only a minor EQE decrease in the fullerene absorption regime which also nicely matches the observation on PDTP-DFBT:PC 61 BM binary solar cells. Again, we observed about no change in the surface topography of the ternary PTB7-Th:PDTP-DFBT:PC ${ }_{61} B M$ layer (Fig. 4c), which indicates that $\mathrm{PC}_{61} \mathrm{BM}$ aggregation is effectively suppressed.

Herein, we have explored in detail the stability of PTB7-Th: PDTP-DFBT:PC ${ }_{61} B M$ bulk-heterojunctions under thermal stress that is typically applied during device lamination $\left(120^{\circ} \mathrm{C}, 2 \mathrm{~h}\right)$. Yet, the solar cells also withstand other annealing processes. Figure S5 in the supplementary information depicts the relative performance of the ternary blends during $200 \mathrm{~h}$ at $85^{\circ} \mathrm{C}, 30 \mathrm{~h}$ at $120^{\circ} \mathrm{C}$ and $30 \mathrm{~min}$ at $150^{\circ} \mathrm{C}$.

We note that PDTP-DFBT can also stabilize other bulkheterojunctions such as the omnipresent PTB7:PC ${ }_{61} B M$, as detailed in Fig. $\mathrm{S} 6$ and Table S2 in the supplementary information.

\section{Domain conformation}

The origin of the thermal stability of the ternary PTB7-Th: PDTP-DFBT:PC ${ }_{61} B M$ solar cells is hidden in the molecular and domain conformation of its components. Since non-destructive measurements are always preferred to probe bulk properties, spectroscopic ellipsometry is one of the methods of choice. Probing the shape of nanodomains in binary bulk-heterojunctions by spectroscopic ellipsometry was previously described by Engmann et al. and exemplified on P3HT:PC ${ }_{61} \mathrm{BM}$ blends. ${ }^{42}$ They employed an effective medium approximation to describe molecular distributions in thin-films by the complex refractive indices $(n, k)$. Following this procedure, we recorded the dielectric functions $\varepsilon$ of the neat components PTB7-Th, PDTP-DFBT and $\mathrm{PC}_{61} \mathrm{BM}$ on glass substrates, and then modeled $\varepsilon$ by fitting $a$ generalized (Gauss) oscillator model to the corresponding data as previously described in the literature. ${ }^{43,44}$ The optical constants ( $n$, $k$ ) of the neat materials were then combined into an effective medium to describe the ternary blend. $k$ essentially represents the absorption of the ternary blends (Fig. S7a, Supp. Information), which is in excellent agreement with the previously discussed thin-film absorption and device EQE (Fig. 3b and Fig. S4, Supp. Information).

The description of such an effective medium strongly depends on the size and shape of the material domains formed by its 

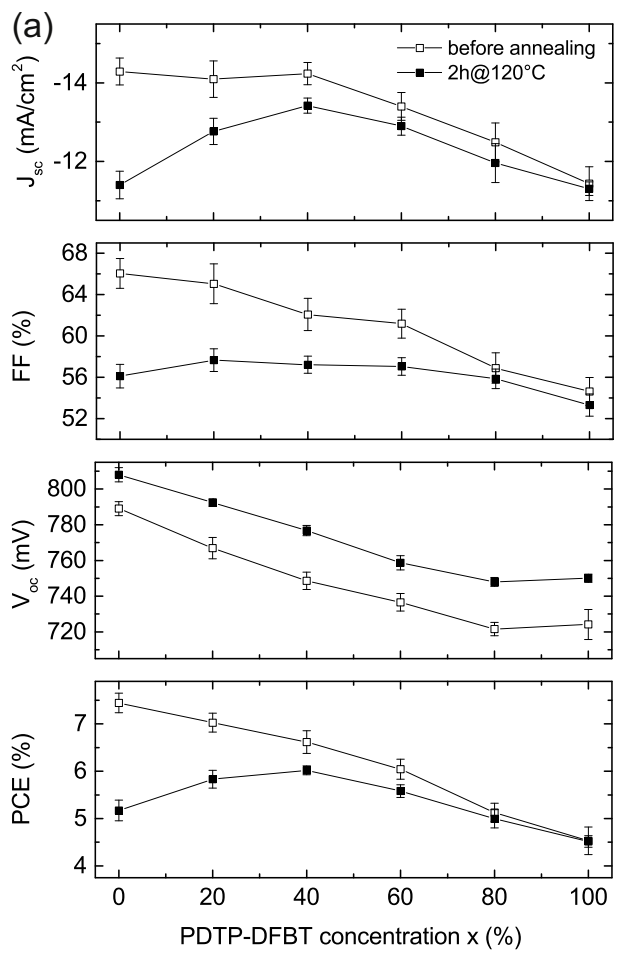
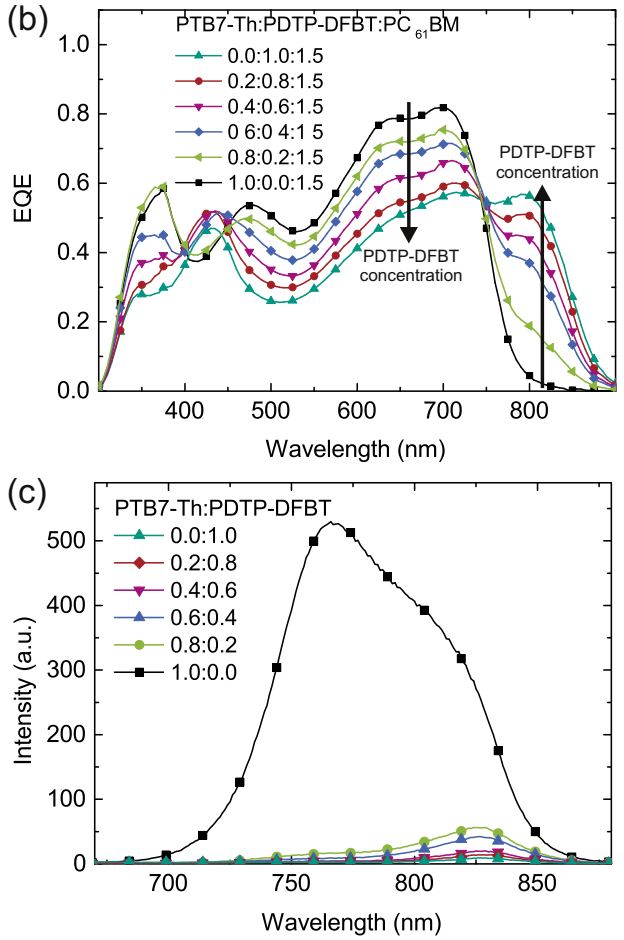

Fig. 3 a Photovoltaic performance of ternary PTB7-Th:PDTP-DFBT:PC ${ }_{61} B M([1-x]: x: 1.5 \mathrm{w} / \mathrm{w} / \mathrm{w})$ solar cells versus PDTP-DFBT concentration $x$ measured under AM1.5 irradiation before and after annealing $\left(120^{\circ} \mathrm{C}, 2 \mathrm{~h}\right)$. b Corresponding external quantum efficiencies. c Photoluminescence spectra of binary PTB7-Th:PDTP-DFBT donor blends excited at $\lambda=610 \mathrm{~nm}$, showing efficient quenching of the PTB7Th photoluminescence by PDTP-DFBT

components. The underlying theory is well described in the literature. ${ }^{45}$ The interaction of domains with the electromagnetic field and hence the effective dielectric function $\varepsilon_{\text {eff }}$ depend on the domains' polarizability. The latter in turn depends on the volume fractions of the components as well as the shape of the domains. According to Bruggeman theory, for two materials, $\varepsilon_{\text {eff }}$ can be calculated by:

$\varepsilon_{\text {eff }}=\frac{\varepsilon_{\mathrm{a}} \varepsilon_{\mathrm{b}}+\kappa \varepsilon_{\mathrm{h}}\left(f_{\mathrm{a}} \varepsilon_{\mathrm{a}}+f_{\mathrm{b}} \varepsilon_{\mathrm{b}}\right)}{\kappa \varepsilon_{\mathrm{h}}+\left(f_{\mathrm{a}} \varepsilon_{\mathrm{b}}+f_{\mathrm{b}} \varepsilon_{\mathrm{a}}\right)}$,

(ref. 43) where $\varepsilon_{\mathrm{a}}$ and $\varepsilon_{\mathrm{b}}$ represent the dielectric functions of the neat materials while $f_{\mathrm{a}}$ and $f_{\mathrm{b}}$ represent the corresponding volume fractions. $\varepsilon_{\mathrm{h}}$ is the dielectric function of the host material, i.e., the surrounding matrix. In Bruggeman theory, the host is the blend of the two materials described by $\varepsilon_{\mathrm{a}}$ and $\varepsilon_{\mathrm{b}}$, i.e, $\varepsilon_{\mathrm{h}}=\varepsilon_{\text {eff }}$ (ref. 46). Equation 1 can be extended to any number of involved materials.

The screening factor $q \in[0,1], k=(1-q) / q$ describes the screening of the electromagnetic field by the two components. We found that $q$ changes when the domain conformation changes. Hence, $q$ is well suited to describe the domain conformation, also in ternary bulk-heterojunctions. We recorded ellipsometric data from PTB7-Th:PDTP-DFBT:PC ${ }_{61} B M$ thin-films comprising different polymer mixing ratios before and after annealing and used $q$ as a modeling parameter. $q$ versus the PDTP-DFBT concentration $x$ is plotted in Fig. 5. At $x=0 \%$, i.e., a binary PTB7-Th:PC ${ }_{61} \mathrm{BM}$ blend, the screening factor $q$ multiplies by a factor of three upon annealing. This indicates a change in domain conformation (order, size or shape) within the bulkheterojunction which is the origin of the PTB7-Th:PC ${ }_{61}$ BM solar cell performance loss during annealing as observed above. On the other hand, the screening factor of binary PDTP-DFBT:PC ${ }_{61} B M$ bulk-heterojunctions $q=0.1 \quad(x=100 \%)$ does not change upon annealing. This can be attributed to a stable domain conformation and is very much in accordance with the stable performance of PDTP-DFBT:PC ${ }_{61} B M$ solar cells before and after annealing. In between both extremes, $q$ changes depending on the PDTP-DFBT concentration $x$. At $x=40 \%$ and beyond, $q$ remains mostly unaffected by thermal annealing, reflecting the enhanced stability of the ternary blends with high PDTP-DFBT content and in particular of the PTB7-Th:PDTP-DFBT:PC ${ }_{61}$ BM $(0.6: 0.4: 1.5 \mathrm{w} / \mathrm{w} / \mathrm{w})$ blend investigated above. We note that the observed changes in PTB7-Th:PC ${ }_{61} B M$ surface topography in Fig. 2c may also stem from the change of domain shapes that is reflected in the screening factor $q$.

\section{CONCLUSION}

In this study, we observed excellent thermal stability of solar cells comprising binary PDTP-DFBT:PC ${ }_{61} \mathrm{BM}$ bulk-heterojunctions, whereas PTB7-Th:PC ${ }_{61} \mathrm{BM}$ solar cells show a $30 \%$ relative performance loss upon annealing at $120^{\circ} \mathrm{C}$ for $2 \mathrm{~h}$. When replacing $40 \mathrm{wt} \%$ of PTB7-Th with PDTP-DFBT, the performance of PTB7-Th:PC ${ }_{61} B M$ solar cells is maintained. At the same time, however, this ternary blend exhibits the enhanced thermal stability of PDTP-DFBT:PC ${ }_{61}$ BM binary blends. A comprehensive spectroscopic ellipsometry study and the interpretation of the screening factor indicate that the ternary blend exhibits a stable domain conformation. This is in accordance with atomic force microscopy investigations. Notably, the annealing conditions investigated herein are also compatible with plastic substrates such as PET.

This leads us to conclude that ternary D-D-A blends comprising two polymers are not only a promising concept for enhancing the PCEs of organic solar cells, but may also be a facile route to devices with improved thermal stability, either on short terms during fabrication (e.g., lamination processes), or on long terms during device operation. 

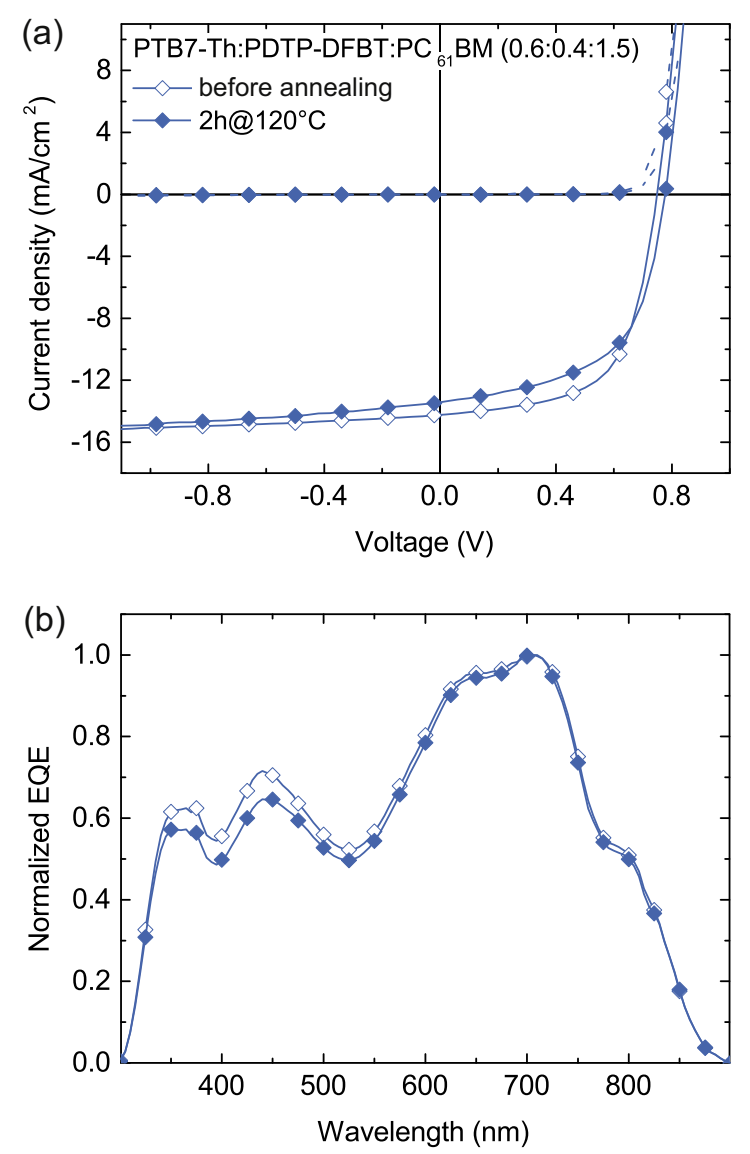

(c)

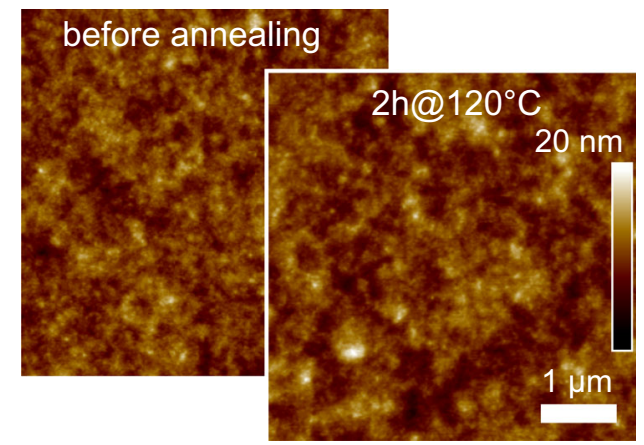

Fig. 4 a Typical $J-V$ curves of PTB7-Th:PDTP-DFBT:PC ${ }_{61} B M$ (0.6:0.4:1.5) solar cells under AM1.5 irradiation (solid lines) and in the dark (dashed lines) before and after thermal annealing $\left(120^{\circ} \mathrm{C}, 2\right.$ h), demonstrating excellent thermal stability of the ternary bulkheterojunction. b The (normalized) corresponding external quantum efficiencies show only minor deterioration in the fullerene absorption regime. c Typical AFM images of PTB7-Th:PDTP-DFBT: $\mathrm{PC}_{61} \mathrm{BM}$ (0.6:0.4:1.5) thin-films before (back) and after annealing (front), qualitatively showing no change in surface topography

\section{METHODS}

\section{Device fabrication}

All organic solar cells were fabricated according to the device architecture depicted in Fig. 1d. Patterned indium tin oxide (ITO, layer thickness $125 \mathrm{~nm}$, $R_{\square}=15 \Omega / \mathrm{sq}$ ) -coated glass substrates were cleaned by sequential ultrasonication in acetone and 2-propanol $(10 \mathrm{~min})$. Afterwards the substrates were transferred into a nitrogen glovebox for the entire fabrication and characterization process. A zinc oxide $(\mathrm{ZnO})$ electron extraction layer was spun from nanoparticle dispersion (Nanograde Ltd., 1 wt $\%$ in 2-propanol, $4000 \mathrm{rpm}, 30 \mathrm{~s}, 30 \mathrm{~nm}$ ) and thermally annealed on a hotplate $\left(80^{\circ} \mathrm{C}, 10 \mathrm{~min}\right)$. Poly[[4,8-bis[5-(2-ethylhexyl)thiophen-2-yl]benzo [1,2-b:4,5-b']dithiophene-2,6-diyl][3-fluoro-2-[(2-ethylhexyl)carbonyl]thieno

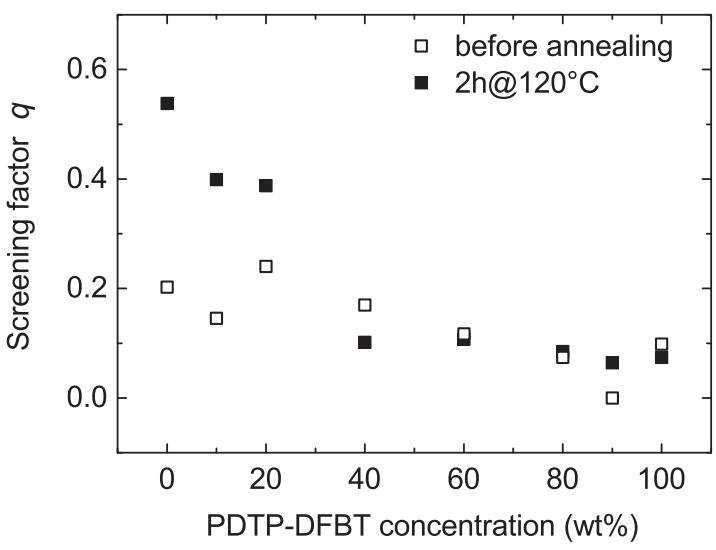

Fig. 5 The evolution of the screening factor $q$ of ternary PTB7-Th: PDTP-DFBT:PC ${ }_{61}$ BM blends versus the PDTP-DFBT concentration $x$ before (open squares) and after annealing (closed squares)

[3,4-b]thiophenediyl] (PTB7-Th, 1-Material Inc., $M_{\mathrm{w}}=108 \mathrm{~kg} / \mathrm{mol}, \bigoplus_{\mathrm{M}}=2.5$ ) and poly[[2,7-(5,5-bis-(3,7-dimethyloctyl)-5H-dithieno[3,2-b:2',3'-d]pyran)alt-4,7-(5,6-difluoro-2,1,3-benzothiadiazole)]] (PDTP-DFBT, 1-Material Inc., $\left.M_{\mathrm{w}}=37 \mathrm{~kg} / \mathrm{mol}, \Theta_{\mathrm{M}}=2.4\right)$ were dissolved in o-xylene with different mass ratios. The overall polymer concentration was kept constant at $10 \mathrm{~g} / \mathrm{L}$. [6,6]phenyl $\mathrm{C}_{61}$-butyric acid methyl ester ( $\mathrm{PC}_{61} \mathrm{BM}$, Solenne, $99 \%$ ) was dissolved separately. The solutions were mixed to yield a polymer:fullerene ratio of $1: 1.5$. The solution was stored overnight on a hotplate at $85^{\circ} \mathrm{C}$. Afterwards, 2 vol\% of the co-solvent $p$-anisaldehyde were added to the stock solution. The photoactive layer was spin cast from the warm solution $(1500 \mathrm{rpm}$, $60 \mathrm{~s}, 100 \mathrm{~nm})$. After initial film drying at room temperature $(10 \mathrm{~min})$, the films were dried on a hotplate at moderate temperature $\left(60^{\circ} \mathrm{C}, 10 \mathrm{~min}\right)$. The molybdenum oxide/silver $\left(\mathrm{MoO}_{x} / \mathrm{Ag}, 10 \mathrm{~nm} / 100 \mathrm{~nm}\right)$ counter electrode was vacuum deposited (base pressure $10^{-6} \mathrm{mbar}$ ) using a shadow mask to define the active area of the solar cell $\left(3 \times 3.5 \mathrm{~mm}^{2}\right)$.

\section{Characterization}

Stability tests of solar cells were performed on non-encapsulated solar cells in inert atmosphere and under continuous heating on a hotplate at $120^{\circ} \mathrm{C}$ for $2 \mathrm{~h}$. Current density-voltage $(J-V)$ curves were recorded with a sourcemeasurement unit (Keithley 238) under illumination from a spectrally monitored solar simulator (Oriel $300 \mathrm{~W}, 1000 \mathrm{~W} / \mathrm{m}^{2}$, ASTM AM $1.5 \mathrm{G}$ ), calibrated by a KG5 filtered silicon reference cell (91150-KG5, Newport). We note that light-soaking did not show any effect on the optoelectronic properties of the solar cells. Layer thicknesses were measured with a tactile stylus profiler (Dektak XT, Bruker). Absorbance and transmittance spectra were recorded using a UV-Vis-NIR spectrophotometer (Cary 5000, Agilent Technologies) equipped with an integrating sphere $(\varnothing=150 \mathrm{~mm})$. PL spectra of films were measured on a fluorescence spectrophotometer (Cary Eclipse, Agilent Technologies) using an excitation wavelength $\lambda=610 \mathrm{~nm}$.

HOMO energies of the semiconductors were estimated from the ionization potential measured by photoelectron yield spectroscopy in air (PESA, AC-2E, Riken Keiki, beam power $10 \mathrm{nW}$ ). The EQE was measured using chopped monochromatic probe light, generated by a high-pressure xenon plasma lamp (450 W LSH601, LOT Oriel) and a monochromator (Omni- $\lambda 300$, LOT Oriel), including a set of wavelength filters (MSZ3122, LOT Oriel) and optics. The solar cell response was measured using a lock-in amplifier (DLPCA-S, Femto Messtechnik GmbH and eLockln 203, Anfatec Instruments $A G$ ) synchronized with the chopped monochromatic probe light. No bias light was applied. The $J_{\mathrm{sc}}$ calculated from the EQE and the $J_{\mathrm{sc}}$ measured under the solar simulator matched within 5\% (PTB7-Th) and 15\% (PDTP-DFBT) range. Atomic force microscope (AFM, Dimension ICON, Bruker) images were recorded on fully functional solar cells next to the top electrodes in tapping mode (TESP tip).

Spectroscopic ellipsometry measurements and data analysis were carried out on a Woollam VASE $^{\circ}$ system with an AutoRetarder and the corresponding WVASE32 Data Acquisition and Analysis Software ${ }^{\odot}$. Data were recorded at wavelengths from $300 \mathrm{~nm}$ to $1600 \mathrm{~nm}$ in steps of $10 \mathrm{~nm}$ and at angles of incidence from $35^{\circ}$ to $75^{\circ}$ in $5^{\circ}$ intervals. Additionally, a transmission spectrum at normal incidence was recorded to validate the obtained thicknesses and imaginary parts of the refractive indices. Since Mueller-Matrix measurements do not show any distinct in-plane 
anisotropy, an isotropic sample type was assumed. All ellipsometry measurements were performed using neat material films on glass at room temperature and under ambient conditions.

\section{Data availability}

The data that support the findings of this study are available from the corresponding author upon reasonable request.

\section{ACKNOWLEDGEMENTS}

The authors acknowledge funding by the German Federal Ministry of Education and Research (BMBF) under contract 03EK3501H (project POPUP). The AFM was made available through funding by the BMBF, grant no. 03EK3504 (Project TAURUS). We acknowledge support by the Center for Functional Nanostructures (CFN). We thank Nanograde Ltd. for supplying zinc oxide nanoparticles.

\section{AUTHOR CONTRIBUTIONS}

D.L., D.F., A.S. and D.B. carried out the experiments of binary and ternary bulk heterojunction solar cells. A.M. and R.D. performed the spectroscopic ellipsometry measurements. T.L. performed the atomic force microscope measurement. A.C. supervised and coordinated the study. All authors discussed the results and jointly wrote the manuscript.

\section{ADDITIONAL INFORMATION}

Supplementary Information accompanies the paper on the npj Flexible Electronics website (https://doi.org/10.1038/s41528-017-0011-z).

Competing interests: The authors declare that they have no competing financial interests.

Publisher's note: Springer Nature remains neutral with regard to jurisdictional claims in published maps and institutional affiliations.

\section{REFERENCES}

1. Liu, Y. et al. Aggregation and morphology control enables multiple cases of highefficiency polymer solar cells. Nat. Commun. 5, 5293 (2014)

2. Green, M. A., Emery, K., Hishikawa, Y., Warta, W. \& Dunlop, E. D. Solar cell efficiency tables (Version 45). Prog. Photovolt. 23, 1-9 (2015).

3. Jørgensen, M. et al. The state of organic solar cells-a meta analysis. Sol. Energy Mater. Sol. Cells 119, 84-93 (2013).

4. Cheng, P. \& Zhan, X. Stability of organic solar cells: challenges and strategies. Chem. Soc. Rev. 45, 2544-2582 (2016).

5. Gevorgyan, S. et al. Lifetime of organic photovoltaics: status and predictions. Adv. Energy Mater. 6, 1501208 (2016)

6. Roberts, S. \& Guariento, N. Building Integrated Photovoltaics: A Handbook (Birkhäuser GmbH, Germany, 2009).

7. Berny, S. et al. Solar trees: first large-scale demonstration of fully solution coated, semitransparent, flexible organic photovoltaic modules. Adv. Sci. 3, 1500342 (2015).

8. Yang, X., van Duren, J. K., Janssen, R. A., Michels, M. A. \& Loos, J. Morphology and thermal stability of the active layer in poly (p-phenylenevinylene)/methanofullerene plastic photovoltaic devices. Macromolecules. 37, 2151-2158 (2004).

9. Yang, X. et al. Nanoscale morphology of high-performance polymer solar cells. Nano Lett. 5, 579-583 (2005).

10. Wong, $\mathrm{H}$. et al. Morphological stability and performance of polymer-fullerene solar cells under thermal stress: the impact of photoinduced PC60BM oligomerization. ACS Nano 8, 1297-1308 (2014).

11. Zhang, C. Overcoming the thermal instability of efficient polymer solar cells by employing novel fullerene-based acceptors. Adv. Energy Mater. 7, 1601204 (2017).

12. Zhao, W. et al. Fullerene-free polymer solar cells with over $11 \%$ efficiency and excellent thermal stability. Adv. Mater. 28, 4734-4739 (2016).

13. Cheng, P. \& Zhan, X. Versatile third components for efficient and stable organic solar cells. Mater. Horiz. 2, 462-485 (2015).

14. Derue, L. et al. Thermal stabilisation of polymer-fullerene bulk heterojunction morphology for efficient photovoltaic solar cells. Adv. Mater. 26, 5831-5838 (2014).

15. Rumer, J. W. et al. Dual function additives: a small molecule crosslinker for enhanced efficiency and stability in organic solar cells. Adv. Energy Mater. 5, 1401426 (2015)

16. Chao, Y. C. et al. Enhanced thermal stability of organic photovoltaics via incorporating triphenylamine derivatives as additives. Sol. Energy Mater. Sol. Cells 157 666-675 (2016)
17. Santo, Y., Jeon, I., Yeo, K. S., Nakagawa, T. \& Matsuo, Y. Mixture of [60] and [70] PCBM giving morphological stability in organic solar cells. Appl. Phys. Lett. 103 073306 (2013).

18. Lindqvist, $C$. Fullerene mixtures enhance the thermal stability of a non-crystalline polymer solar cell blend. Appl. Phys. Lett. 104, 153301 (2014).

19. Cheng, P. et al. Alloy acceptor: Superior alternative to PCBM toward efficient and stable organic solar cells. Adv. Mater. 28, 8021-8028 (2016).

20. Cheng, Y. J., Hsieh, C. H., Li, P. J. \& Hsu, C. S. et al. Morphological stabilization by in situ polymerization of fullerene derivatives leading to efficient, thermally stable organic photovoltaics. Adv. Funct. Mater. 21, 1723-1732 (2011).

21. Diacon, A. et al. Cross-linkable azido C 60 -fullerene derivatives for efficient thermal stabilization of polymer bulk-heterojunction solar cells. J. Mater. Chem. $C$ 2, 7163-7167 (2014).

22. Deb, N. et al. Thermo-cross-linkable fullerene for long-term stability of photo voltaic devices. J. Mater. Chem. A 3, 21856-21863 (2015).

23. Sivula, K., Ball, Z. T., Watanabe, N. \& Fréchet, J. M. Amphiphilic diblock copolymer compatibilizers and their effect on the morphology and performance of polythiophene: fullerene solar cells. Adv. Mater. 18, 206-210 (2006).

24. Tsai, J. H. et al. Enhancement of P3HT/PCBM photovoltaic efficiency using the surfactant of triblock copolymer containing poly (3-hexylthiophene) and poly (4vinyltriphenylamine) segments. Macromolecules 43, 6085-6091 (2010).

25. Ferenczi, T. A. et al. Organic semiconductor: insulator polymer ternary blends for photovoltaics. Adv. Mater. 23, 4093-4097 (2011).

26. Liao, S. H., Jhuo, H. J., Cheng, Y. S. \& Chen, S. A. Fullerene derivative-doped zinc oxide nanofilm as the cathode of inverted polymer solar cells with low-bandgap polymer (PTB7-Th) for high performance. Adv. Mater. 25, 4766-4771 (2013).

27. He, Z. et al. Single-junction polymer solar cells with high efficiency and photovoltage. Nat. Photonics 9, 174-179 (2015).

28. Dou, L. et al. Synthesis of $5 \mathrm{H}$-dithieno $\left[3,2-b: 2^{\prime}, 3^{\prime}-d\right]$ pyran as an electron-rich building block for donor-acceptor type low-bandgap polymers. Macromolecules 46, 3384-3390 (2013).

29. Zhang, S. et al. Improved photon-to-electron response of ternary blend organic solar cells with a low band gap polymer sensitizer and interfacial modification. J. Mater. Chem. A 4, 1702-1707 (2016).

30. Dkhil, S. B. et al. Toward high-temperature stability of PTB7-based bulk heterojunction solar cells: impact of fullerene size and solvent additive. Adv. Energy Mater. 7, 1601486 (2016)

31. Sprau, C. et al. Highly efficient polymer solar cells cast from non-halogenated xylene/anisaldehyde solution. Energy Environ. Sci. 8, 2744-2752 (2015).

32. Müller, C. On the glass transition of polymer semiconductors and its impact on polymer solar cell stability. Chem. Mater. 27, 2740-2754 (2015).

33. Lee, T. H. et al. Investigation of charge carrier behavior in high performance ternary blend polymer solar cells. Adv. Energy Mater. 6, 1600637 (2016).

34. Yang, L., Zhou, H., Price, S. C. \& You, W. Parallel-like bulk heterojunction polymer solar cells. J. Am. Chem. Soc. 134, 5432-5435 (2012).

35. Zhang, M., Zhang, F., Wang, J., An, Q. \& Sun, Q. Efficient ternary polymer solar cells with a parallel-linkage structure. J. Mater. Chem. C 3, 11930-11936 (2015).

36. Lu, L., Chen, W., Xu, T. \& Yu, L. High-performance ternary blend polymer solar cells involving both energy transfer and hole relay processes. Nat. Commun. 6, 7327 (2015).

37. Zhang, J. et al. Conjugated polymer-small molecule alloy leads to high efficient ternary organic solar cells. J. Am. Chem. Soc. 137, 8176-8183 (2015).

38. Street, R. A., Davies, D., Khlyabich, P. P., Burkhart, B. \& Thompson, B. C. Origin of the tunable open-circuit voltage in ternary blend bulk heterojunction organic solar cells. J. Am. Chem. Soc. 135, 986-989 (2013).

39. Kouijzer, S., Li, W., Wienk, M. M. \& Janssen, R. A. Charge transfer state energy in ternary bulk-heterojunction polymer-fullerene solar cells. J. Photonics Energy $\mathbf{5}$ 057203-057203 (2015).

40. Khlyabich, P. P., Burkhart, B. \& Thompson, B. C. Compositional dependence of the open-circuit voltage in ternary blend bulk heterojunction solar cells based on two donor polymers. J. Am. Chem. Soc. 134, 9074-9077 (2012).

41. Khlyabich, P. P., Rudenko, A. E., Street, R. A. \& Thompson, B. C. Influence of polymer compatibility on the open-circuit voltage in ternary blend bulk heterojunction solar cells. ACS Appl. Mater. Interfaces 6, 9913-9919 (2014).

42. Engmann, S., Turkovic, V., Gobsch, G. \& Hoppe, H. Ellipsometric investigation of the shape of nanodomains in polymer/fullerene films. Adv. Energy Mater. 1, 684-689 (2011).

43. Fujiwara, H. Spectroscopic Ellipsometry: Principles and Applications (John Wiley \& Sons, Ltd, UK, 2007).

44. Klein, M. F., de Medeiros, G. Q. G., Kapetana, P., Lemmer, U. \& Colsmann, A Modeling approach to derive the anisotropic complex refractive index of polymer: fullerene blends for organic solar cells utilizing spectroscopic ellipsometry. J. Photonics Energy 5, 057204-057204 (2015).

45. Aspnes, D. E. Optical properties of thin films. Thin Solid Films 89, 249-262 (1982). 
46. Woollam, J. A. Guide to Using WVASE32 software for Spectroscopic Ellipsometry Data Acquisition and Analysis (J.A. Woollam Co., USA 2002).

(c) Open Access This article is licensed under a Creative Commons Attribution 4.0 International License, which permits use, sharing, adaptation, distribution and reproduction in any medium or format, as long as you give appropriate credit to the original author(s) and the source, provide a link to the Creative Commons license, and indicate if changes were made. The images or other third party material in this article are included in the article's Creative Commons license, unless indicated otherwise in a credit line to the material. If material is not included in the article's Creative Commons license and your intended use is not permitted by statutory regulation or exceeds the permitted use, you will need to obtain permission directly from the copyright holder. To view a copy of this license, visit http://creativecommons. org/licenses/by/4.0/.

(c) The Author(s) 2017 